\title{
Estrategias de motivación para el incremento de la satisfacción laboral en empresas de servicios de alimentos
}

Motivation strategies to increase job satisfaction in food services companies

\author{
Yesenia Mignini \\ yemp_67@hotmail.com \\ ORCID: 0000-0002-8898-4758
}

\author{
Geisser Sánchez \\ geissersanchez@gmail.com \\ ORCID: 0000-0001-8942-5918
}

María Dávila

ucfaces05@gmail.com

ORCID: 0000-0003-4917-7643

Pastichísimo, C.A. - Valencia - Venezuela

Artículo recibido febrero 2020 / Arbitrado en marzo 2020 / Aceptado junio 2020 / Publicado en julio 2020

RESUMEN

El objetivo del estudio es analizar las estrategias de motivación que incrementen el nivel de satisfacción de los empleados de la empresa Pastichisimo. Esta investigación fue sustentada por una variedad de teorías como la de los dos factores de Herzberg, las necesidades de MacClelland y el enfoque gerencial del Empowerment. Dicha investigación se abordó bajo un tipo de estudio de campo, del tipo descriptivo de acuerdo con el nivel de conocimiento que se alcanzó, así mismo el estudio se apoyó en una investigación documental. Los resultados analizados permitieron concluir que los empleados se encuentran desmotivados y esto afecta directamente a la productividad de la institución, por lo cual es necesario la elaboración de dichas estrategias para la organización, ya que sus empleados necesitan sentirse motivados, para lograr incrementos en la productividad y la atención de manera positiva y satisfactoria de sus clientes.

Palabras clave: Motivación, Estrategias, Satisfacción Laboral
The objective of the study is to design motivation strategies that increase the level of satisfaction of the employees of the company Pastichisimo. This research was supported by a variety of theories such as Herzberg's two factors, MacClelland's needs, and the Empowerment management approach. This research was addressed under a type of field study, of the descriptive type according to the level of knowledge reached, and the study was supported by documentary research. The analyzed results allowed to conclude that the employees are unmotivated and this directly affects the productivity of the institution, which is why it is necessary to elaborate these strategies for the organization, since its employees need to feel motivated to achieve increases in productivity. and the positive and satisfactory attention of its clients.

Keywords: Motivation, Strategies, Job Satisfaction 


\section{INTRODUCCIÓN}

El capital humano es uno de los principales elementos de las empresas, por ello las organizaciones han de asumir compromisos de gestión sensibles a las necesidades de sus trabajadores. Tomando en consideración lo anteriormente descrito, la presente investigación constituye una aproximación de estrategias que incrementen la satisfacción laboral en empresas del sector de servicios de alimentos, tomándose como referencia para este estudio a la empresa Pastichisimo, C.A.

En el caso específico de esta organización, ubicada en Valencia, estado Carabobo, dedicada a la elaboración, venta y distribución de pizzas y pastichos que es su línea directa de preparación, les convendría la implementación de dichas estrategias, debido a que incrementaría la motivación laboral de sus empleados y esto garantizaría alcanzar las metas de la organización.

La motivación es todo aquello que nos impulsa seguir adelante, para alcanzar nuestras metas y todo lo que deseamos en la vida. Según Mazzetti, V. (2013) "la motivación es lo que hace que un individuo actúe y se comporte de una determinada manera. Es una combinación de procesos intelectuales, fisiológicos y psicológicos que decide, en una situación dada". Lo expresado por el autor implica a que la motivación es el factor base emocional para el ser humano y para cualquier profesional, estar motivado indica realizar las tareas cotidianas sin apatía y sin un sobreesfuerzo adicional.

En el ámbito laboral es la capacidad que tienen las organizaciones para mantener el estímulo positivo de sus empleados realizando ajustes o adaptaciones necesarias de acuerdo a los intereses organizacionales, el hecho de manejar información sobre como guiar a los trabajadores hacia desempeños sobresalientes, puede orientar las iniciativas respecto a cómo, a través de la motivación se pueden lograr los objetivos empresariales.

Es importante señalar que la motivación juega un papel primordial en el impulso del ser humano, lo que tiene como base un conjunto de necesidades de diversa índole que el trabajador experimenta y que pueden ser satisfechas mediante su vínculo laboral. Por consiguiente, es una fuerza interna que mueve a las personas a realizar una acción con el objeto de obtener una satisfacción.

En este sentido a veces resulta difícil distinguir entre la motivación y la satisfacción laboral, debido a su estrecha relación. El nivel de satisfacción en el trabajo depende de la comparación entre lo que tengo y el impulso de conseguir lo que quiero, esto depende de numerosos factores como el ambiente físico donde trabaja, el sentido de logro o realización que le procura el trabajo, la posibilidad de aplicar sus conocimientos, así como a su desarrollo intelectual asumir retos.

$\mathrm{Se}$ puede señalar entonces que la satisfacción representa una interacción entre los empleados y su ambiente laboral, en donde se busca la congruencia entre lo que los empleados quieren de su trabajo y lo que los empleados sienten que reciben.

La productividad, la motivación y la satisfacción laboral están estrechamente ligadas, lo que lleva necesariamente a la afirmación de que si hay motivación necesariamente hay productividad; debido a que las personas cuando se sienten a gusto y satisfechos con su trabajo, la productividad suele mejorar, trayendo los tan deseados beneficios económicos.

\section{Aspectos teórico}

En el mundo empresarial la motivación es un factor clave para incrementar y mantener la productividad del recurso humano, aparentemente resulta sencillo pensar que las 
personas se sienten a gusto en el ambiente laboral en el que se desempeñan, pero la realidad demuestra que mantener al personal motivado es un proceso complejo. Este aspecto ha sido analizado y estudiado desde diferentes perspectivas, atribuyendo a la motivación diversos significados y conclusiones.

\section{Teorías de la motivación}

Desde hace muchos años grandes filósofos han aportado teorías que agrupan cantidades de enfoques sobre la motivación laboral. Como la teoría de la jerarquía de necesidades de Abraham Maslow (1943), el cual observa como las necesidades humanas ascienden desde el nivel más bajo al más alto, en forma de jerarquía, siendo las necesidades fisiológicas la base de la pirámide; esta teoría afirma que las necesidades de orden superior no pueden ser satisfechas mientras las necesidades básicas o de orden inferior no hayan sido cubiertas.

Las Teorías de $\mathrm{X}-\mathrm{Y}$ de Douglas McGregor (1960): contrapone dos teorías, la teoría X y la teoría $\mathrm{Y}$. La teoría $\mathrm{X}$, tiene una visión pesimista para el ser humano, entendiendo $q$ a las personas no les gusta trabajar, y si pueden no lo harán. Para que lo hagan habrá que presionarlas, controlarlas o amenazarlas, además los trabajadores evitan asumir responsabilidades. La teoría $Y$, es una visión optimista de las personas, partiendo de que a los empleados si les gusta trabajar, se auto controlan, asumen responsabilidades y son capaces de tomar decisiones creativas.

Es importante señalar, según la teoría del autor ya mencionado que no siempre es bueno recurrir al trabajo obligado o bajo presión, ni mucho menos utilizar el método de la amenaza o la de hostigar a un empleado para que este realice sus funciones. Allí no está la solución, por lo tanto, se caería en el grave error de tener trabajadores desanimados y sin ganas de hacer sus funciones por los males ya descritos, se debe de aplicar estrategias inteligentes que cambie paradigma en cuanto a la visión que debe de tener los trabajadores en relación a su trabajo, que vaya de la mano con las ganas y la intención de realizar bien sus labores, logrando día a día estar más cerca del logro de la empresa.

\section{Teoría de la motivación e higiene de Herzberg (1959)}

En cuanto a la teoría de los dos factores de Frederick Herzberg también conocida como bifactorial (Motivación e Higiene) los factores de motivación están bajo el control del individuo ya que estos están relacionados con la satisfacción en el cargo y con la naturaleza de las tareas que ejecuta, y los de higiene están fuera del control de las personas, debido a que están relacionados con la insatisfacción, pues se localizan en el ambiente que rodean a los sujetos y abarcan las condiciones en que desempeñan su trabajo.

Esta teoría parte de la idea de que la satisfacción-no satisfacción está estrechamente relacionado o vinculado con las tareas diarias que realiza cada trabajador tales como, el crecimiento personal del individuo, el reconocimiento profesional, la responsabilidad de cada persona, y todos aquellos aspectos de autorrealización que cada empleado desempeña. También comenta que los cargos y las actividades que se realizan solo están creados para observar la eficacia y alcances económicos que eso generan, sin observar más allá al empleado, limitando de manera directa las oportunidades de creatividad que cada trabajador pueda tener; es, por ende, que el desinterés influencia la desmotivación, porque la organización solo ofrece un lugar para trabajar y no un lugar para que los empleados puedan desarrollar todas sus habilidades. 
Otros de los aspectos que destaca el autor en su teoría, es que el factor insatisfacción- no insatisfacción, depende de manera directa de los factores extrínsecos de higiene o ergonómico, como el ambiente o el entorno que lo rodea y la manera o forma como desempeñar el trabajo, estos sin duda, son indicadores motivacionales $y$ que no depende de cada empleado, sin embargo, nos comenta el autor que reduce la insatisfacción. Entre los principales factores están, el tipo de dirección o supervisión que las personas $u$ empleados reciben, las políticas de las empresas, relaciones interpersonales, reglamentos técnicos y las condiciones de trabajo.

Esta teoría nos da las bases necesarias para tener más conocimiento de las necesidades que poseen los trabajadores, puesto que cada uno de ellos tienen necesidades distintas, por consiguiente, podemos tomarlas en cuenta para diseñar las estrategias necesarias para que se sientan más a gusto en su entorno laboral y así la motivación se incremente.

Los factores de desarrollo o motivadores intrínsecos al trabajo son: realización, el trabajo mismo, responsabilidad y progreso o desarrollo. Los factores que llevan a evitar la insatisfacción o factores de higiene extrínsecos al trabajo incluyen: la política y la administración, supervisión, relaciones interpersonales, condiciones de trabajo, salario, nivel laboral y seguridad vigentes en la empresa. La teoría de la Higiene-Motivación sugiere que el trabajo debe enriquecerse para conseguir una eficaz utilización del personal. El enriquecimiento de la tarea ofrece al empleado la oportunidad de desarrollarse psicológicamente... este artículo se limita a sugerir aquellos principios y medidas prácticas que se deducen de diversos experimentos realizados con éxito en la industria.

\section{Teoría de las necesidades de McClelland (1961)}

David McClelland (logro, poder y afiliación); estas se van adquiriendo en el trascurso de la vida a través de copiar los comportamientos de otros individuos, sin embargo, no hay dos personas que las tengan exactamente en las mismas prioridades. Por ejemplo, una persona tendrá una gran necesidad de logro, pero poca necesidad de afiliación. Otra podría tener una alta necesidad de afiliación, pero poca necesidad de poder.

Estas necesidades son aprendidas, por lo que la organización puede condicionar conductas favorables al rendimiento a través de reconocimientos vinculados directamente sobre esa conducta, ya que la motivación se activa mediante estímulos que inducen a la persona a pensar que una determinada conducta laboral va a satisfacer esa necesidad. Las diversas observaciones sobre la motivación laboral, sobre las variables que motivan e incentivan a las personas a llevar a cabo una tarea. Como lo indica la teoría antes expuesta, claramente es importante tener en cuenta cada una de las necesidades de los trabajadores, tanto de logro, poder y afiliación, para que así estas puedan aportar el mejor rendimiento de los mismos en sus actividades.

\section{Enfoque gerencial del Empowerment}

Mosley, Megginson y Pietri (2005), hacen referencia a un enfoque gerencial comúnmente utilizado en la actualidad, el Empowerment, como el proceso de compartir el poder con los empleados, aumentando la confianza para desempeñar el trabajo, siendo vital la enseñanza del individuo a percibir el correcto significado de sus actividades, sintiéndose competente, y capaz de elegir las tareas de trabajo, teniendo gran 
impacto en las decisiones estratégicas de la institución.

La realización de la estrategia Empowerment ayuda a mejorar la comunicación y el trato de los gerentes con los empleados, existiendo una retroalimentación de información porque no solo las ideas del gerente son las correctas, los empleados también pueden tener ideas potenciales que vayan en pro al logro de esos objetivos, mejorando la relación y la confianza de parte y parte.

Navarro, Arrieta y Vicente (2008) señalan que existen teorías contemporáneas sobre la motivación, tal es el caso del Modelo de Hackman y Oldman, para el diseño de puestos, caracterizado por las habilidades y la retroalimentación, para que las personas experimenten estados psicológicos asociados con el significado de su trabajo, la responsabilidad del mismo y la eficiencia en el desempeño de sus tareas, este modelo evidencia una relación de correlación entre las actividades en el puesto de trabajo que conlleva al desarrollo personal y el impacto en el desarrollo psicológico del individuo.

Estas teorías de diferentes enfoques motivacionales son de gran provecho en los procedimientos para la resolución de problemas, con este tipo de inconvenientes como lo es la insatisfacción laboral a causa de la falta de motivación de los trabajadores, ya que influyen en destacar lo importante que son las metas individuales, la comunicación, la retroalimentación, la equidad y la afinidad que debe existir tanto como en el entorno laboral y la tarea que realice el individuo dentro de la organización, donde todo debe estar en concordancia con las metas del grupo, por lo que es lógico pensar en el desempeño basado en el trabajo en equipo para lograr el éxito en las instituciones.
Existen diversas técnicas motivacionales especiales, entre las que se mencionan las siguientes y más comunes: Dinero: Este nunca puede ser pasado por alto como motivador, los administradores y economistas le dan un alto sitio al dinero en la escala de los motivadores, mientras que los científicos tienden a restarle importancia. Participación: Se refiere a que se le consulte al empleado respecto a acciones que lo afectan, es decir, que se le tome en cuenta. La Participación es crucial como motivador en una organización ya que se le da una oportunidad al empleado de expresar sus incomodidades y la gerencia al conocerlas, trabajar en función a ellas. Calidad de vida laboral: Consiste en un enfoque de sistemas de puestos y un prometedor avance en el amplio terreno del enriquecimiento del puesto.

Dentro de los Modelos Motivacionales tenemos el Modelo de Expectativa, este es el más conocido de estos modelos. Este modelo tiene sienta sus bases en las investigaciones realizadas por Tolman y Lewin, pero se asocia con investigadores contemporáneos como Vroom, Lawler, Hackman y Porter. La Teoría de las expectativas sostiene que los individuos como seres pensantes y razonables, tienen creencias y abrigan esperanzas y expectativas respecto a eventos futuros en sus vidas. Por lo que para analizar la motivación se requiere conocer lo que las personas buscan de la organización y cómo creen poder obtenerlo

Otro elemento importante a considerar es la satisfacción laboral. Henderson (2011) "señala que la satisfacción laboral está muy influenciada por lo que está pasando en el resto de la vida de las personas". En este sentido podemos afirmar que, si alguien no está satisfecho en otros aspectos de su existencia, afectará la capacidad de encontrar satisfacción en el trabajo y esta insatisfacción afectará también otras áreas de su 
vida, transformándose en un círculo vicioso de la infelicidad.

No cabe duda que el estado anímico y emocional de las personas guarda relación con el entorno en que este se desenvuelva, si un individuo se encuentra con problemas emocionales sin duda alguna esta persona no se encontrara deseoso de cumplir con entusiasmo su jornada, por lo general esta desmotivación personal la arrastrara también a su desenvolvimiento laboral y conllevaría a otro problema para el individuo.

Según Colquitt et al (2007) la satisfacción laboral se define como un agradable estado emocional que resulta de la evaluación de un trabajo o la experiencia de un trabajo. Representa cómo la persona se siente acerca de su trabajo y lo que piensa acerca de éste. Según el autor, la satisfacción laboral está fuertemente relacionada con la satisfacción en la vida, siendo la satisfacción laboral un fuerte predictor de esta última. En ese contexto, señala que, si queremos sentirnos mejor en la vida, tenemos que encontrar la forma de sentirnos más satisfechos en el trabajo.

Es importante señalar que existe una relación directa entre la vida del individuo y que perspectiva tiene este en relación a su trabajo. Ambos van de la mano con la persona, los dos están íntimamente vinculados, si existe alguna afectación de uno se visualiza también en el otro entorno.

En otro orden de ideas algunos meta-análisis han encontrado que la satisfacción laboral tiene una influencia significativa en la abstención en el trabajo, rotación, desempeño laboral y estrés, así como también se ha revelado que la insatisfacción laboral es el mejor predictor de las rotaciones (Eslami, 2012). De forma similar, Colquitt et al (2007) plantea que trabajos simples y rutinarios tienden a disminuir la satisfacción laboral a la vez que aumentan las abstenciones y rotaciones. Y en la misma línea, Eslami et al (2012) indica que la satisfacción laboral puede afectar e influenciar la productividad en el trabajo, la rotación de los trabajadores y su retención.

Tomando en cuenta la información de los autores antes mencionados, es de total importancia señalar que la satisfacción laboral es garante del desarrollo organizacional de una empresa y el logro de sus objetivos propuestos, conocer que la monotonía en la ejecución de sus funciones y la poca rotación de cargos, afecta el desenvolvimiento en el rol y las tareas de los empleados, la monotonía sin duda alguna repercute en la ejecución de sus labores y los desmotiva ya que estos, no realizan sus funciones con total entusiasmo sino como el día a día que a cada empleado les toca realizar para obtener una remuneración a cambio de su rutina diaria.

\section{MATERIALES Y METODO}

El presente estudio se sustenta en una investigación de campo de tipo descriptivo con apoyo documental. Se consideró encuestar a todos los trabajadores de la empresa Pastichísimo, C.A., ubicada en Valencia, Venezuela, dedicada a la preparación y venta de comidas preparadas, siendo esta muestra de 20 personas. La encuesta aplicada estuvo conformada por 15 preguntas de respuestas politómicas con cuatro alternativas disponibles.

Los datos obtenidos se analizaron mediante métodos cualitativos y cuantitativos, lo cual permitió conocer y evidenciar la necesidad de la aplicación de técnicas y estrategias motivacionales por parte de la empresa Pastichisimo, para mejorar el rendimiento laboral de sus trabajadores. 


\section{RESULTADOS Y DISCUSIÓN}

En la encuesta aplicada, los trabajadores de la empresa Pastichísimo, C.A., fueron consultado acerca de diversos aspectos relacionados con la remuneración, satisfacción laboral, beneficios no salariales, motivación, ambiente laboral, relaciones interpersonales, comunicación, crecimiento personal, acciones de la gerencia, liderazgo y trabajo en equipo.

Como producto de ello, se pudo evidenciar, la carencia de aplicación de estrategias que promuevan y fomenten la satisfacción laboral, consiguiendo como protagonista el objetivo fundamental de dicha investigación. Según los resultados, la empresa no desarrolla programas motivacionales que puedan de alguna $u$ otra forma inducir a los trabajadores a tener una conducta determinada en pro de mejorar sus trabajos. Koontz y Weirich (2008).

En función al análisis realizado, se evidencia la carencia de motivación que limita la satisfacción laboral en sus empleados. Por otra parte, se observó que la empresa no posee un plan de formación ni capacitación que ayude a los trabajadores a desarrollarse y a crecer individualmente en el ámbito laboral y eso coadyuva a la necesidad de querer surgir profesionalmente sin el deseo de querer de asumir nuevas responsabilidades y proyectos. Es por ello que se debe facilitar la creación de un conjunto de estrategias que vayan en pro al incremento de la motivación, la existencia de una mejor comunicación entre la alta gerencia y los empleados y, por último, proponer a la gerencia preocuparse y ocuparse por el crecimiento personal de cada uno de los empleados que laboran allí, todo con la finalidad de mejorar y preservar la calidad de servicio de la empresa estudiada. A partir de lo anterior, se presentarán unas estrategias para su posible aplicación.

En la actualidad el éxito de las organizaciones para lograr ser competitivas, lo determina la calidad de sus recursos humanos, por ello se considera el recurso más importante. Los trabajadores proveen a las organizaciones de las habilidades, la creatividad, el esfuerzo intelectual y físico necesario para contribuir a que la organización lleve a cabo sus operaciones con calidad de servicio y logre alcanzar los objetivos. En este sentido, las organizaciones necesitan del desempeño del recurso humano, para lograr el éxito de los mercados, mediante la producción y mejora de sus servicios, y por lo tanto, la motivación del personal debe ser el objetivo primordial de toda organización que desee alcanzar niveles altos de competitividad y este nunca debe de estar fuera del ambiente empresarial.

La motivación para el trabajador no solo involucra la parte monetaria sino también el ambiente donde se desenvuelven diariamente, las relaciones interpersonales, la interacción con los demás, así como políticas que contribuyan a incentivarlos mediante beneficios sociales como recompensas y reconocimientos en retribución al desempeño de su trabajo; lo que desarrolle su motivación y aumente su autoestima comprometiéndolo con la empresa, como parte importante, dispuesto a dar lo mejor de sí, para que esta, mediante la labor de su personal, obtenga los beneficios deseados.

Dicho esto, debemos inferir en la importancia que tiene que los empleados tengan el conocimiento de la visión y misión y también sobre el poder que tiene el compartirla. Recordemos que la visión es aquella proyección a mediano o largo plazo que la empresa quiere lograr. La visión es el "qué queremos llegar a ser", señalando a un punto deseable que se quiere alcanzar en un lapso, y la misión es una declaración o manifestación duradera del objeto, propósito o razón de ser de una empresa.

Cuando los trabajadores desconocen la visión y la misión de la empresa, todo su 
potencial laboral se enfoca solamente en su realización personal, es decir, en alcanzar sus propios ideales; el empleado trabaja en pro de buscar su propio beneficio y vive al día, porque probablemente sus esfuerzos estén radicados solo en la retribución económica y no en el beneficio que como parte empresa se pueda conseguir. Entonces encontramos dos elementos muy importantes. Motivación: Es tarea de la empresa, dar a conocer la visión de la empresa a sus trabajadores. Esto crea una identidad, luego una motivación que les permita mirar "juntos al mismo norte" y Productividad: Es resultado de la motivación, ya que el empleado afina su trabajo para realizar labores optimizadas, buscando la visión de la empresa sin descuidar la suya propia. Por lo tanto, esto representa grandes beneficios para la compañía.

Finalmente, entre los resultados de la investigación se plantean un conjunto de elementos, referentes a la necesidad de elaborar un plan operativo de estrategias motivacionales, cuyo objetivo es el desempeño laboral de los trabajadores en la empresa Pastichísimo, C.A., todo ello a través de la optimización de la comunicación dentro de la organización, el reforzamiento de las habilidades $y$ conocimientos a los trabajadores acordes al puesto de trabajo, mejorar la interacción con los clientes e innovar incorporando nuevos productos y personalizando los menús.

Este Plan debería enfocarse en estrategias motivacionales para incrementar la satisfacción laboral en dicha organización. Las estrategias que adopte la empresa, motivarán al trabajador y servirán como incentivo, al percibir que su esfuerzo es reconocido y retribuido no solo en dinero sino en actividades que la empresa le ofrezca, basadas en el reconocimiento y que vayan en función al crecimiento personal, la recreación y el esparcimiento.
Un empleado motivado, que se siente a gusto trabajando, aumenta su rendimiento, atiende bien al público: además no se limita a realizar solo las labores correspondientes a su cargo, sino también promueve otras en busca del mejoramiento de la empresa, es decir, participa más en la búsqueda de los objetivos y puede aportar nuevas ideas.

Toda empresa que en su presupuesto incluya el desarrollo de programas de capacitación, dará a conocer a sus empleados el interés que tiene en ellos como personas, como trabajadores, como parte importante de esa organización.

La capacitación cuenta con objetivos muy claros, entre los cuales podemos mencionar: conducir a la empresa a una mayor rentabilidad y a los empleados a tener una actitud más positiva, mejorar el conocimiento del puesto a todos los niveles, elevar la moral de la fuerza laboral ayudar al personal a identificarse con los objetivos de la empresa y obtener una mejor imagen. La forma en que cada miembro de una empresa se comunica e interactúa con cada uno de sus clientes, genera impresiones e impacta de manera positiva o negativa en el proceso de generación de valor de toda la compañía. Por esa razón, es importante comprender y tomar conciencia de que siempre es posible mejorar la calidad de atención a los clientes, a su vez, todos somos responsables de atenderlos bien.

\section{CONCLUSIONES}

Una empresa exitosa, es aquella que tiene bien claro sus objetivos económicos, sociales y organizacionales; aquella que es capaz de observarse y medirse interna y externamente, aquella que enfrenta los resultados y busca soluciones a la problemática detectada y el cual cuenta con una visión clara, con una imagen transparente de lo que quiere conseguir y en lo que quiere convertir la institución en el tiempo. Se puede decir entonces que las empresas 
exitosas se caracterizan por un alto sentido de pertenencia, un alto grado de motivación en todos los niveles, y una gran capacidad de liderazgo.

Toda empresa que quiera alcanzar el éxito debe tener claro, que la principal diferencia con la competencia está en su capital humano. Por lo tanto, constantemente debe ofrecer a sus empleados oportunidades de desarrollo, esquemas que los impulsen a colaborar y tener un equilibrio con su vida personal, técnicas para mantenerlos motivados y compensaciones justas.

Los incentivos, los beneficios sociales, el entusiasmo y la energía son solo algunas de las cosas que se requiere para realizar la mayor parte del trabajo con esmero y motivación.

REFERENCIAS

Colquitt (2011). Revista de Psicología Aplicada Copyright de la Asociación Americana de Psicología. Justicia organizacional y estrés: el papel mediador de Conflicto trabajo-familia. Universidad de Florida. Herzberg (1959) Teoría de los dos factores, Juan Manso Pinto,
El Legado de Frederick Irving Herzberg. Revista Universidad EAFIT, Universidad EAFIT Medellín, Colombia

Koontz y Weihrich (2009), Administración: Una perspectiva global, México: McGraw-Hill, $12^{\circ}$ ed.

Maslow (1943), teoría sobre la motivación humana. Psychological Review.

Mazzetti. (2013), artículo de la motivación como estrategia en las empresas.

McGregor (1960), El lado humano de las organizaciones, en la MIT SloanSchool of Management.

McClelland (1961). Teoría Logro, poder y afiliación. Nueva York: Van Nostrand.

Mosley, Megginson y Pietri (2005), Supervisión: la práctica del Empowerment, desarrollo de equipos de trabajo y su motivación, Editorial: INTERNATIONAL Thomson Editores, México. $6^{\mathrm{a}}$ ed.

Navarro, Arrieta y Vicente (2008), factores asociados a la emergencia de patrones diferenciales de la motivación en el trabajo psicothema Vol. $20 \quad \mathrm{~N}^{\circ} 4$, universidad de Oviedo. España. México. 